\title{
AN INSIGHT INTO FACIAL MASK AND SOCIAL DISTANCE MONITORING SYSTEM BASED ON DEEP LEARNING OBJECT DETECTOR TO PREVENT COVID-19 TRANSMISSION
}

\author{
Iram Javed, \\ Samina Khalid, \\ Tehmina Shehryar, \\ Yasir Mehmood*, \\ Iftikhar Ahmad \\ Department of CS\&IT, \\ Mirpur University of Science \\ and Technology (MUST), \\ Mirpur, AJK, Pakistan
}

\begin{abstract}
:
During the COVID-19 pandemic, facial mask detection and monitoring the social distance between persons is an essential and challenging task. Along with other several machine learning techniques, deep learning has been successfully applied for object detection. In this paper, we have thoroughly explored the deep learning object detection methods for facial masks and physical distance. We have discussed an overview of object detection methods in the form of taxonomy and analyse one-stage and two-stage object detectors. In the end, some open research challenges have been presented as well.
\end{abstract}

Keywords:

COVID-19, Deep Learning, Machine Learning, Social Distance, Object Detection.
Correspondence:

Yasir Mehmood

e-mail:

yasir.mehmood@must.edu.pk

\section{INTRODUCTION}

COVID-19 is an infectious disease caused by the SARS-CoV-2 (2019$\mathrm{nCoV}$ ) virus, first identified in Wuhan city of China on 29 December 2019 [1]. Due to its worldwide infections in millions of people, the World Health Organization (WHO) declared it as a pandemic in March 2020. In this response, governments have imposed wearing masks, social distancing, and increasing awareness of hygiene. It is a respiratory disease and the reproductive ratio of corona virus is greater than other acute respiratory problems like SARS and MERS [2,3]. Therefore, for safety some precautions measures needed like washing hand regularly, wearing a face mask, and maintaining social distance between people [4].

The deep learning object detection model is divided into two types, the first type is one-stage detector including SSD [5] and YOLO [6] and the other is a two-stage detector including R-CNN model [7] and Faster R-CNN model [8]. These detectors have gained tremendous success in the field of machine learning to overcome corona virus. Therefore, it is necessary to take precautionary measurements like wearing a facial mask and keeping social distances. Therefore, facial mask detection and social distance systems have many applications in the domain of machine learning. 
The facial mask detection system is closely related to object detection techniques that identify whether a mask is present on the face or not and recognize the location of the face. Face detection system achieved high accuracy and performance with deep learning SSD refinement face detector namely Refine Face [9]. Correct wearing face mask with classifier SRCNET applied deep learning detector and achieved accuracy $98.70 \%$ [10].

Artificial intelligence is a powerful way to predict and fight against COVID-19.AI and deep learning technique help to monitor social distance by using fixed monocular camera and thermal image with detector Faster R-CNN and YOLOv4 [11,12]. Another social distance maintenance problem scenario has been provided statistics during violation with Social Signal Processing methods based on deep learning. Estimation of Visual distance measurement is automatic and also maintains privacy policy but needs attention to work more on the effectiveness and ethical process of visual social distance system [13]. YOLO v3 has been applied for social distance monitoring with deep learning bounding boxes of object detection and frames in video display index violation [14]. In another research [15], object detectors handle facial mask detection, social distance monitoring, and measure body temperature based on the Internet of things (IoT)by using OpenCV Harcascade classifier. Faster R-CNN and SSD indicated that ensuring proper use of face masks and maintain social distance to restrict virus growth [16-18]. An automated monitoring system has been effectively worked against the fight of controlling respiratory disease COVID-19. There have been broad research aspects of the abovementioned era of corona virus. To limit the scope, we mainly study (2015-2021) facial masks and social distance measuring deep learning detectors representing the research contributions. To the best of our knowledge, no taxonomy has been presented related to deep learning detectors for facial masks and measure social distance in the existing literature. In literature, we propose taxonomy to give a summary of the evolution in the area of deep learning detectors in Section 2. Based on the in-depth exploration, research challenges have been upraised in Section 3. Lastly, the discussion is concluded in Section 4.

\section{TAXONOMY}

Corona virus has caused the deaths of millions of people all over the world. In this epidemic, the best precautionary measures are facial masks and maintaining social distance. In the literature, several object detection models have been proposed to automate and improve the efficiency of facial masks and measure the social distance between persons. These models are categorized into (i) one-stage detector and (ii) two-stage detector, as shown in Figure 1.

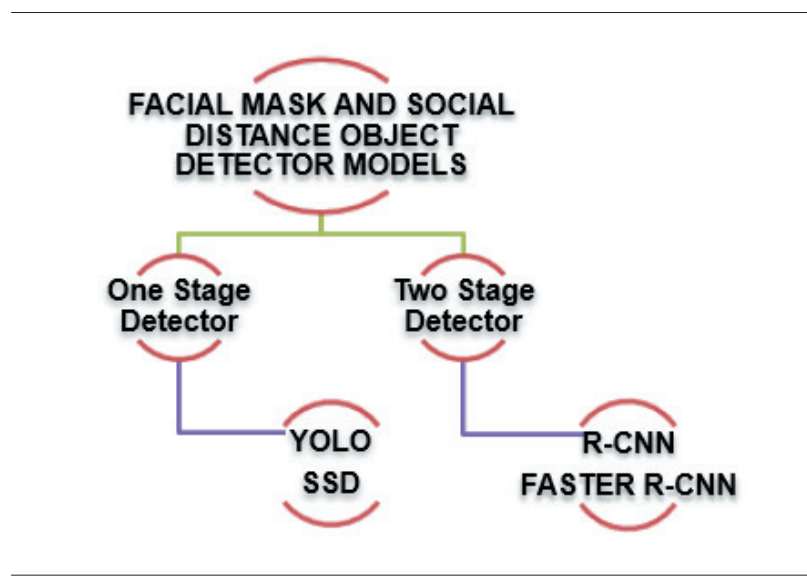

Figure 1- Taxonomy of Facial Mask and Social Distance Object Detector Model

\subsection{ONE-STAGE DETECTOR}

One-stage object detectors belong to supervised machine learning classification and regression methods helpful to detect facial masks and measure social distance. The algorithm runs clearly and concisely distributed into training and testing. Generally, testing directly alters the input data into output image data and makes a resultant detection frame over decoding. On the other hand, the labelled data encodes during training to create constant with the consistent output data of $\mathrm{CNN}$ and compute loss. One-stage detectors are YOLO [6], SSD [5].

\subsubsection{YOLO}

You Only Look Once (YOLO), a well-known deep learning detector, introduced by Redmon et al. [6] in 2016, uses a fully convolution network. The core concept is to treat the detector as a regression problem, using a feature extractor network that reduces false detection and reads 45 frames per second in real life. In the literature, various versions of the YOLO detector have been presented to automate the monitoring system of facial masks and measure social distance. Loey et al. [18] have proposed Yolo-v2 based on feature extractor network ResNet-50 to annotate and detect medical face masks. Besides, the author has applied mean IoU to the evaluation number of the anchor box and achieved $81 \%$ 
average precision by using Adam optimizer. However, CUDA is used for GPU learning with MATLAB, Tensorflow, and Deep learning libraries. The author also observed a deep learning detector to solve classification problems in the technical field.

In this regard, Said et al. [19] proposed the Pynq-YOLO-Net light-weighted Convolutional Neural Network with YOLO framework to detect face masks. Moreover, the system has been executed on Pynq Z1 embedded quantized acquired 94.6\% Precision and 95.8\% Recall. Based on computation, the face mask detection system used feature extraction on hardware devices, and results were performed on software. To tackle the binary classification problem, the author eliminates the computation of class probability and YOLO calculates confidence scores of predicted bounding boxes and increases the speed of the detector. Similarly, NS Punn et al. [14] have presented a Deepsort technique YOLO v3 to check COVID-19 social distance between persons. The author also conducted a comparison with state-of-art detector Faster RCNN and SSD consider classification and localization with frames per second, mean average precision, and loss values.

2.1.2. SSD

Single shot detectors (SSD) based on COVID-19 monitoring system methods have been investigated for multiple categories. The detector contains a VGG16 network that directly predicts box offset and scores of bounding boxes for fix set in a feature map. Therefore, each feature map contains different scales of the default bounding box. Likewise, for different feature maps, a ratio of bounding boxes has been calculated with regular space among the lowest and highest layer. After that, matched bounding boxes compare with ground truth to enhance the accuracy and track the principle of the feed-forward convolutional neural network [14].

In another research work, Deng et al. [20] have projected an improved facial mask detection method based on SSD. To prove the value of the presented solution, feature fusion and attention learning are used to determine a person wearing a mask or not. That is why we have obtained $91.7 \%$ accuracy and built a channel domain of attention procedure with a feature map. However, there are still some problems needed to be set, among these, the author used a small dataset that provided low performance. Hence the largest COCO dataset used by S Neelavathy Pari et al. [21] to monitor social distance in smart phone applications and cameras. The SSD model handles Conv5-3 beside the VGG-16 layer that extracts the feature map. Ahamad et al. [22] detect a person in a region of interest using image processing, computer vision, and Single Shot Multibox Detector MobileNet. The distance has been calculated between people to be detected in the image and then associated with static pixels. The distance was scaled between the central points and the overlying boundary among peoples in the segmented tracking part. The author also worked on a violation system to generate alerts by using python.

\subsection{TWO-STAGE DETECTOR}

The two-stage detector belongs to the family of RCNN. It is divided into two stages. The first step filters out Region of Interests (ROI) from an image by extracted feature. ROIs contain possible boxes in an image. The second step, utilize ROIs and features to calculate class probabilities and final bounding boxes. Therefore, to attain an optimum trade-off among accuracy and speed these types of detector are useful like R-CNN detector [23] Faster R-CNN detector [24].

\subsubsection{R-CNN}

It is a deep learning object detector that proved effective and well-organized based on region. The author Girshick [25] presented a Region Convolutional Neural Network detector that works on $\mathrm{CNN}$ model on dataset PASCAL VOC having advanced detection performance [26] using HOG-like features. Region Convolutional Neural Network detectors have four parts. The initial part produces class-independent region proposals. Each region proposal extracts the feature vector in the second part. The third part has a set of class exact linear Support vector machines to classify objects. The last part has a regression bounding box for exactly bounding box prediction.

A larger amount of dataset used by fine-tuning during the pre-training phase on the definite dataset is a better training way for $\mathrm{CNN}$ to attain faster convergence. However, Girshick firstly pre-train the deep convolutional neural networks on a larger dataset. The latter fully connected layer has been changed by the CNN ImageNet. The next phase has utilized stochastic gradient descent (SGD) toward modification of CNN constraints taking the twisted windows proposal. Finally, the classification layer has a fully connected layer with the $(\mathrm{N}+1)$ method. 


\subsubsection{FASTER R-CNN}

This type of detector contains two networks with the R-CNN family, first is the Region proposal network (RPN) that detects objects by creating region and network proposals. Region proposal network positions box of the region (named as anchors) and intends having objects. Secondly, it generated features to get object features by using $\mathrm{CNN}$. The classification layer helpful to predict class and the regression layer provide bounding box coordinates more accurately. Region proposal used a selective search algorithm based on CPU, which precedes about $2 \mathrm{~s}$ per image. Faster R-CNN not only carries the region proposal period from $2 \mathrm{~s}$ to 10 s however also permits the proposal region step to divide layers [8]. In another research, Singh et al. [27] applied Faster $\mathrm{R}-\mathrm{CNN}$ and YOLO v3 to detect face masks. The resNet101-FPN architecture is used as a backbone during the training phase of the model. The author proposed a method around the person's face to draw bounding boxes, check that mask present on the person's face, and keep record data of face masks of the person every day. Singh has also associated the performance of Faster RCNN and YOLO v3 models.

\section{OPEN RESEARCH CHALLENGES}

\subsection{SYSTEM FOOLED BY PLACING A HAND ON MOUTH AND NOSE}

In literature, facial mask detection techniques along with their limitations have been discussed comprehensively. The deep learning-based facial mask technique can detect a face covered with a mask or not but the system can be confusing when placing a hand on the nose and mouth [32]. In another research, only front images of faces have been shown accurate results. The system cannot detect face masks when the right and left sides are seen [28]. So, researchers need to fill this research gap.

\subsection{LIMITED DATASET}

With the evolution in the era of deep learning, detectors based on facial masks and social distance systems require a large amount of data to maintain performance and accuracy. Until recently, limited datasets available to train and test models $[15,36]$. In the existing literature, a new object detector model MASK-R-CNN is not used in the area of the COVID-19 prevention method.
Deep Learning CNN algorithms utilized to optimize the facial mask system model. However, scholars have not introduced agent-based modeling (ABM) for facial mask detection systems to confirm the effects of the applied optimization techniques. Moreover, the ABMbased methods can play a major role to detect human face masks and measure social distance.

\subsection{LACK OF REAL-TIME IMPLEMENTATION}

From the tables $[11,16,22,34]$, it can be shown that $80 \%$ of the proposed deep learning facial mask and social distance control system are based on simulations that do not determine the same applicability in the realtime environment. One of the major problems in realworld implementation is that these methods depend upon complex mathematical models that do not achieve well in the real world [37].

\section{CONCLUSIONS}

In this paper, we have presented taxonomy for automated facial masks detection and social distance with a deep learning object detector system. These systems can handle two types of detector i.e. one-stage detector and two-stage detector. These detectors, based on SSD, YOLO, R-CNN and Faster R-CNN, are discussed along with objectives of each technique. After that performance of these systems are compared and the research gap in the existing literature has been highlighted.

\section{REFERENCES}

1. D. S. Hui et al., "The continuing 2019-nCoV epidemic threat of novel coronaviruses to global health - The latest 2019 novel coronavirus outbreak in Wuhan, China," International Journal of Infectious Diseases, vol. 91. 2020, doi: 10.1016/j. ijid.2020.01.009.

2. M. Olaniyan, M. Muhibi, and T. Ojediran, "Covid 19: Transmission, case fatality rate, protective measures, laboratory diagnosis, and possible laboratory features," Matrix Sci. Medica, vol. 4, no. 3, p. 64, 2020, doi: 10.4103/mtsm.mtsm_6_20.

3. D. Benvenuto, M. Giovanetti, A. Ciccozzi, S. Spoto, S. Angeletti, and M. Ciccozzi, "The 2019-new coronavirus epidemic: Evidence for virus evolution," J. Med. Virol., vol. 92, no. 4, pp. 455-459,2020, doi:10.1002/jmv.25688. 
4. R. Singh and R. Adhikari, "Age-structured impact of social distancing on the COVID-19 epidemic in India," pp. 1-9, 2020,[Online].Available:http://arxiv. org/abs/2003.12055.

5. W. Liu et al., "SSD: Single shot multibox detector," Lect. Notes Comput. Sci. (including Subser. Lect. Notes Artif. Intell. Lect. Notes Bioinformatics), vol. 9905 LNCS, pp. 21-37, 2016, doi: 10.1007/978-3319-46448-0_2.

6. J. Redmon, S. Divvala, R. Girshick, and A. Farhadi, "You only look once: Unified, real-time object detection," Proc. IEEE Comput. Soc. Conf. Comput. Vis. Pattern Recognit., vol. 2016-December, pp. 779-788, 2016, doi: 10.1109/CVPR.2016.91.

7. R. Girshick, "Fast R-CNN," Proc. IEEE Int. Conf. Comput. Vis., vol. 2015 International Conference on Computer Vision, ICCV 2015, pp. 1440-1448, 2015, doi: 10.1109/ICCV.2015.169.

8. S. Ren, K. He, R. Girshick, and J. Sun, "Faster RCNN: Towards Real-Time Object Detection with Region Proposal Networks," IEEE Trans. Pattern Anal. Mach. Intell., vol. 39, no. 6, pp. 1137-1149, 2017, doi: 10.1109/TPAMI.2016.2577031.

9. S. Zhang, C. Chi, Z. Lei, and S. Z. Li, "RefineFace: Refinement Neural Network for High Performance Face Detection," IEEE Trans. Pattern Anal. Mach. Intell., vol. XX,no.X, pp. 1-1,2020, doi:10.1109/ tpami.2020.2997456.

10. Q. Bosheng and D. Li, "Identifying Facemask-Wearing Condition Using Image Super-Resolution with Classification Network," Mdpi Sens., pp. 1-23, 2020.

11. D. Yang, E. Yurtsever, V. Renganathan, K. A. Redmill, and Ü. Özgüner, "A Vision-based Social Distancing and Critical Density Detection System for COVID-19," 2020, [Online]. Available: http://arxiv. org/abs/2007.03578.

12. W. Naudé, "Artificial intelligence vs COVID-19: limitations, constraints and pitfalls," AI Soc., vol. 35, no. 3, pp. 761-765, 2020, doi: 10.1007/s00146020-00978-0.

13. M. Cristani, A. Del Bue, V. Murino, F. Setti, and A. Vinciarelli, "The Visual Social Distancing Problem," IEEE Access, vol. 8, pp. 126876-126886, 2020, doi: 10.1109/ACCESS.2020.3008370.

14. N. S. Punn, S. K. Sonbhadra, S. Agarwal, and G. Rai, "Monitoring COVID-19 social distancing with person detection and tracking via fine-tuned YOLO v3 and Deepsort techniques," pp. 1-10, 2020, [Online]. Available: http://arxiv.org/abs/2005.01385.

15. N. Petrovic and D. Kocic, "IoT-based System for COVID-19 Indoor Safety Monitoring," IcETRAN 2020, no. September,2020,[Online].Available:https://www. researchgate.net/publication/343231422_IoT_based_ System_for_COVID19_Indoor_Safety_Monitoring.
16. M. Razavi, H. Alikhani, V. Janfaza, B. Sadeghi, and E. Alikhani, "An Automatic System to Monitor the Physical Distance and Face Mask Wearing of Construction Workers in COVID-19 Pandemic," 2021, [Online]. Available: http://arxiv.org/abs/2101.01373

17. S. Yadav, "Deep Learning based Safe Social Distancing and Face Mask Detection in Public Areas for COVID-19 Safety Guidelines Adherence," Int. J. Res. Appl. Sci. Eng. Technol., vol. 8, no. 7, pp. 13681375, 2020, doi: 10.22214/ijraset.2020.30560.

18. M. Loey, G. Manogaran, M. H. N. Taha, and N. E. M. Khalifa, "Fighting against COVID-19: A novel deep learning model based on YOLO-v2 with ResNet-50 for medical face mask detection," Sustain. Cities Soc., vol. 65, no. October 2020, p. 102600, 2021, doi: 10.1016/j.scs.2020.102600.

19. Y. Said, "Pynq-YOLO-Net: An embedded quantized convolutional neural network for face mask detection in COVID-19 pandemic era," Int. J. Adv. Comput. Sci. Appl., vol. 11, no. 9, pp. 100-106, 2020, doi: 10.14569/IJACSA.2020.0110912.

20. H. Deng, J. Zhang, L. Chen, and M. Cai, "Improved Mask Wearing Detection Algorithm for SSD," J. Phys. Conf. Ser., vol. 1757, no. 1, 2021, doi: 10.1088/1742-6596/1757/1/012140.

21. S. Neelavathy Pari, B. Vasu, and A. V Geetha, "Monitoring Social Distancing by Smart Phone," Glob. J. Comput. Sci. Technol. C Softw. Data Eng., vol. 20, no. 2, 2020.

22. A. H. Ahamad, N. Zaini, and M. F. A. Latip, "Person Detection for Social Distancing and Safety Violation Alert based on Segmented ROI," Proc. - 10th IEEE Int. Conf. Control Syst. Comput. Eng. ICCSCE 2020, no. August, pp. 113-118,2020,doi: 10.1109/ ICCSCE50387.2020.9204934.

23. L. Jiao et al., "A survey of deep learning-based object detection," IEEE Access, vol. 7, pp. 128837-128868, 2019, doi: 10.1109/ACCESS.2019.2939201.

24. S. Ren, K. He, R. Girshick, and J. Sun, "Faster RCNN: Towards Real-Time Object Detection with Region Proposal Networks," IEEE Trans. Pattern Anal. Mach. Intell., vol. 39, no. 6, pp. 1137-1149, 2017, doi: 10.1109/TPAMI.2016.2577031.

25. R. Girshick, J. Donahue, T. Darrell, J. Malik, U. C. Berkeley, and J. Malik, "1043.0690," Proc. IEEE Comput. Soc. Conf. Comput. Vis. Pattern Recognit., vol. 1, p. 5000, 2014, doi: 10.1109/CVPR.2014.81.

26. Everingham "The pascal visual object classes (voc) challenge. International journal of computer vision," 2010.

27. S. Singh, U. Ahuja, M. Kumar, K. Kumar, and M. achdeva, "Face mask detection using YOLOv3 and faster R-CNN models: COVID-19 environment," Multimedia Tools and Applications, pp. 1-16, 2021. 
28. Z. Wang et al., "Masked Face Recognition Dataset and Application," pp. 1-3, 2020, [Online]. Available: http://arxiv.org/abs/2003.09093.

29. K. S. D. S, A. Rengarajan, and N. K. H. S, "Detecting of Face Mask," no. 12, pp. 132-134, 2020.

30. M. Jiang, X. Fan, and H. Yan, "RetinaMask: A Face Mask detector," 2020, [Online]. Available: http:// arxiv.org/abs/2005.03950.

31. C. Jagadeeswari and M. U. Theja, "Performance Evaluation of Intelligent Face Mask Detection System with various Deep Learning Classifiers Keywords :," Int. J. Adv. Sci. Technol., vol. 29, no. 11, pp. 3074-3082, 2020.

32. M. M. Rahman, M. M. H. Manik, M. M. Islam, S. Mahmud, and J. H. Kim, "An automated system to limit COVID-19 using facial mask detection in smart city network," IEMTRONICS 2020 - Int. IOT, Electron. Mechatronics Conf. Proc., 2020, doi: 10.1109/IEMTRONICS51293.2020.9216386.

33. I. Ghodgaonkar, S. Chakraborty, V. Banna, S. Allcroft, M. Metwaly, F. Bordwell, et al., "Analyzing Worldwide Social Distancing through Large-Scale Computer Vision," arXiv preprint arXiv:2008.12363, 2020.

34. I. Ahmed, M. Ahmad, J. J. Rodrigues, G. Jeon, and S. Din, "A deep learning-based social distance monitoring framework for COVID-19," Sustainable Cities and Society, vol. 65, p. 102571, 2021

35. S. Saponara, A. Elhanashi, and A. Gagliardi, "Implementing a real-time, AI-based, people detection and social distancing measuring system for Covid-19," Journal of Real-Time Image Processing, pp. 1-11, 2021.

36. P. Khandelwal, A. Khandelwal, S. Agarwal, D. Thomas, N. Xavier, and A. Raghuraman, "Using Computer Vision to enhance Safety of Workforce in Manufacturing in a Post COVID World," pp. 1-7, 2020, [Online]. Available: http://arxiv.org/ abs/2005.05287.

37. S. V. Militante and N. V. Dionisio, "Deep Learning Implementation of Facemask and Physical Distancing Detection with Alarm Systems," Proceeding 20203 rd Int. Conf. Vocat. Educ. Electr. Eng. Strength. Framew. Soc. 5.0 through Innov. Educ. Electr. Eng. Informatics Eng. ICVEE2020,2020, doi:10.1109/ ICVEE50212.2020.9243183 


\begin{tabular}{|c|c|c|c|c|}
\hline Author & Method & Objective & Performance & Limitation \\
\hline$[28]$ & $\begin{array}{l}\text { Single shoot } \\
\text { detector }\end{array}$ & $\begin{array}{l}\text { Proposed largest dataset } \\
\text { of masked faces for } \\
\text { detection and recognition }\end{array}$ & $\begin{array}{c}\text { Achieve } 95 \% \text { accuracy } \\
\text { with multi granularity } \\
\text { model }\end{array}$ & $\begin{array}{l}\text { only front side face } \\
\text { images collected for } \\
\text { dataset from internet }\end{array}$ \\
\hline$[29]$ & $\begin{array}{l}\text { Single shoot } \\
\text { detector }\end{array}$ & $\begin{array}{c}\text { Face mask detection in } \\
\text { image/video }\end{array}$ & $\begin{array}{l}\text { Accomplish accuracy by } \\
\text { tuning hyper-parameters }\end{array}$ & $\begin{array}{l}\text { Difficult to detect faces } \\
\text { in crowd }\end{array}$ \\
\hline$[30]$ & $\begin{array}{c}\text { R-CNN } \\
\text { Faster R-CNN }\end{array}$ & $\begin{array}{l}\text { Enhanced ability of face } \\
\text { mask detection with } \\
\text { single shot RetinaNet } \\
\text { detector and feature } \\
\text { pyramid network }\end{array}$ & $\begin{array}{c}\text { Obtain accuracy } 2.3 \% \& \\
1.5 \% \text { greater than } \\
\text { base-line for precision, } \\
11.0 \% \text { and } 5.9 \% \text { greater } \\
\text { than base-line for recall. }\end{array}$ & $\begin{array}{l}\text { ResNet backbone not } \\
\text { work with other } \\
\text { component due to limited } \\
\text { dataset performance }\end{array}$ \\
\hline$[31]$ & $\begin{array}{l}\text { Single Shoot } \\
\text { detector }\end{array}$ & $\begin{array}{l}\text { Compare deep learning } \\
\text { classifier with } \\
\text { optimization algorithm } \\
\text { to check performance of } \\
\text { face mask detection }\end{array}$ & $\begin{array}{c}\text { ADAM has high accuracy } \\
\text { as compared to ADAGRAD } \\
\text { and SGD MobileNetV2 } \\
\text { classifier also high } \\
\text { accuracy during testing }\end{array}$ & $\begin{array}{c}\text { Absence of alert system } \\
\text { And social distance } \\
\text { measurement, security } \\
\text { issues }\end{array}$ \\
\hline$[32]$ & $\begin{array}{c}\text { Deep learning } \\
\text { Convolutional } \\
\text { Neural Network }\end{array}$ & $\begin{array}{l}\text { Facial mask detection in } \\
\text { smart city through CCTV } \\
\text { camera, in violation case } \\
\text { inform to smart city } \\
\text { authority }\end{array}$ & 98.7\% Achieve accuracy & $\begin{array}{l}\text { Confuse system with } \\
\text { hand covered face, } \\
\text { incorrectly identify } \\
\text { person location on } \\
\text { vehicle. }\end{array}$ \\
\hline$[15]$ & $\begin{array}{l}\text { OpenCV library, } \\
\text { ontology }\end{array}$ & $\begin{array}{l}\text { IoT based system to } \\
\text { detect face mask, check } \\
\text { social distance and sense } \\
\text { temperature without } \\
\text { contact }\end{array}$ & $\begin{array}{c}\text { Mask detection accuracy } \\
84-91 \% \text {, distancing check } \\
65-73 \% \text {, temp. Sensing } \\
0.5-2.5^{\circ} \mathrm{C}\end{array}$ & $\begin{array}{c}\text { Low frame rate in image/ } \\
\text { video, Missing efficient } \\
\text { security and mask } \\
\text { allocation }\end{array}$ \\
\hline$[14]$ & YOLO v3 & $\begin{array}{l}\text { Monitoring real time } \\
\text { social distance }\end{array}$ & $\begin{array}{l}\text { YOLO v3 with deep-sort } \\
\text { acquire better result as } \\
\text { compared to mAP and } \\
\text { FPS }\end{array}$ & $\begin{array}{c}\text { Video frames } \\
\text { violation occurrence are } \\
\text { not recorded, } \\
\text { Privacy issue }\end{array}$ \\
\hline$[11]$ & $\begin{array}{c}\text { Faster R-CNN and } \\
\text { YOLOv4 }\end{array}$ & $\begin{array}{l}\text { Artificial intelligence } \\
\text { detect real time social } \\
\text { distance and critical } \\
\text { density }\end{array}$ & $\begin{array}{l}\text { Accuracy and } \\
\text { performance are good to } \\
\text { monitor social distance }\end{array}$ & $\begin{array}{c}\text { Do not record real time } \\
\text { data, crowd analysis still } \\
\text { a challenge }\end{array}$ \\
\hline$[33]$ & $\begin{array}{l}\text { YOLO } \\
\text { Single Shoot } \\
\text { detector }\end{array}$ & $\begin{array}{l}\text { Crowd counting and } \\
\text { social distance measure }\end{array}$ & Robust performance & $\begin{array}{c}\text { Incorrectly detected } \\
\text { image of person on banner }\end{array}$ \\
\hline$[34]$ & YOLOv3 & $\begin{array}{c}\text { Social distance } \\
\text { monitoring and track } \\
\text { violation }\end{array}$ & $\begin{array}{l}\text { Accuracy with transfer } \\
\text { learning } 98 \%, \text { without } \\
\text { 92\% and tracking model } \\
\text { 95\% accuracy }\end{array}$ & $\begin{array}{l}\text { Improve model for } \\
\text { Indoor and outdoor } \\
\text { environment in future. }\end{array}$ \\
\hline$[35]$ & YOLOv2 & $\begin{array}{l}\text { Indoor and outdoor } \\
\text { environment AI system } \\
\text { for social distance } \\
\text { classification and } \\
\text { measuring, also body } \\
\text { tempt analysis with } \\
\text { thermal camera }\end{array}$ & $\begin{array}{l}\text { high accuracy and low } \\
\text { computational power }\end{array}$ & $\begin{array}{l}\text { Newly YOLOv4 use in } \\
\text { future, 2D parameters } \\
\text { used only to detect } \\
\text { person, not 3D }\end{array}$ \\
\hline$[22]$ & $\begin{array}{l}\text { Single shoot } \\
\text { detector }\end{array}$ & $\begin{array}{c}\text { Indoor and outdoor } \\
\text { social distance with } \\
\text { feature based segmented } \\
\text { ROI alert system }\end{array}$ & $\begin{array}{c}\text { Outdoor testing accuracy } \\
\text { level } 56.6 \%-60 \% \text {, indoor } \\
100 \%\end{array}$ & $\begin{array}{l}\text { Outdoor detection is } \\
\text { difficult }\end{array}$ \\
\hline
\end{tabular}




\begin{tabular}{|c|c|c|c|c|}
\hline$[36]$ & $\begin{array}{l}\text { Single shoot } \\
\text { detector }\end{array}$ & $\begin{array}{l}\text { Social distancing, face } \\
\text { mask detection to } \\
\text { maintain safe } \\
\text { environment }\end{array}$ & $\begin{array}{l}\text { MobileNetV2 model } \\
94.1 \% \text { face mask accuracy } \\
\text { and social distance is } \\
\text { more than } 90 \% \text { accuracy }\end{array}$ & $\begin{array}{l}\text { Limited feature data, } \\
\text { Partially hidden face by a } \\
\text { person create model } \\
\text { confusion, missing } \\
\text { contactless attendance }\end{array}$ \\
\hline$[16]$ & Faster R-CNN & $\begin{array}{l}\text { Face covered with mask } \\
\text { and social distance for } \\
\text { road construction worker }\end{array}$ & $\begin{array}{c}\text { Accuracy Face mask } \\
\text { detection } 90 \%\end{array}$ & $\begin{array}{l}\text { Only reliable for the } \\
\text { Four road construction } \\
\text { worker. }\end{array}$ \\
\hline$[37]$ & $\begin{array}{l}\text { Single shoot } \\
\text { detector }\end{array}$ & $\begin{array}{c}\text { Face mask and } \\
\text { physical distance with } \\
\text { alarm system }\end{array}$ & accuracy rate of $97 \%$ & $\begin{array}{l}\text { Do not detect face mask } \\
\text { and physical distance at } \\
\text { the same time, require } \\
\text { high performance in } \\
\text { detecting and recognizing }\end{array}$ \\
\hline$[17]$ & $\begin{array}{l}\text { Single shoot } \\
\text { detector }\end{array}$ & $\begin{array}{l}\text { Face mask and social } \\
\text { distance detection and } \\
\text { generate alert signal }\end{array}$ & $\begin{array}{c}\text { Obtain accuracy } 85 \% \\
\text { and } 95 \%\end{array}$ & $\begin{array}{c}\text { Not analysis coughing } \\
\text { and sneezing detection } \\
\text { non-available }\end{array}$ \\
\hline
\end{tabular}

Table 1 - Literature Review 